\title{
MOVIMENTI SOCIALI E GLOBALIZZAZIONE
}

\author{
di Donatella della Porta e Hanspeter Kriesi
}

\section{Introduzione}

Nel corso dell'ultimo decennio, gli studiosi dei movimenti sociali negli Stati Uniti ed in Europa hanno prestato sempre più attenzione al contesto politico nel quale essi si mobilitano. In questo processo, la ricerca non solo ha fatto sempre più riferimento alla scienza politica per completare le sue concezioni originali (principalmente fornite dalla sociologia, dalla storia e dalla economia), ma è divenuta anche più comparata, focalizzandosi sull'impatto dei contesti politici nazionali, regionali e locali sulla mobilitazione e sulle sue conseguenze in vari paesi. Con la comparazione cross-nazionale, l'attenzione si è diretta agli effetti del cambiamento nel contesto internazionale sui sistemi sociali e sulla politica a livello nazionale. In altre parole, la ricerca sui movimenti sociali è divenuta lentamente consapevole che la divisione tra la politica comparata e le relazioni internazionali è sempre più anacronistica. Anche nello studio dei movimenti sociali, la sfida «è combinare i risultati di ambedue le prospettive senza perdere di vista i loro singoli contributi» (Garrett e Lange 1995, 654). È quello che cercheremo di fare nel corso di questo articolo, concentrandoci sull'impatto delle crescenti interazioni tra contesti politici nazionali ed internazionali e movimenti sociali in un mondo sempre più globale.

Quello di globalizzazione è un concetto alla moda, usato per definire cose diverse. Nel nostro lavoro l'idea di base è che, nel mondo contemporaneo, l'azione sociale in un tempo e in un luogo dati è condizionata in modo crescente da azioni sociali che si svolgono in luoghi da essa molto distanti. Come suggerisce Giddens $(1990,64)$, globalizzazione implica la creazione e l'intensificazione di «relazioni sociali di estensione mondiale che collegano località distinte in modo tale che eventi locali sia- 
no plasmati da eventi che accadono a molta distanza e viceversa». Se questo processo di interdipendenza crescente su scala globale ha origini più lontane nel tempo (Wallerstein 1990), la rivoluzione tecnologica degli anni ottanta e gli sviluppi economici, culturali e politici ad essa conseguenti hanno contribuito ad una intensificazione «sia della realtà della interdipendenza globale sia della consapevolezza del mondo come un tutt'uno» (Robertson 1992, 8). La rivoluzione nelle comunicazioni trasforma in modo crescente il mondo in un villaggio globale, dove ci raggiungono instantaneamente $\mathrm{i}$ messaggi inviati dai luoghi più distanti. Nel sistema culturale la sostanza della modernità occidentale, promossa dalle scienze e dalle professioni così come dall'industria del tempo libero, ha facilitato la diffusione su scala globale dei valori e delle credenze occidentali (Strang e Meyer 1993, 500 e ss.). Nel sistema economico, un'interdipendenza crescente porta il trasferimento della produzione a paesi dove i salari sono più bassi, alla creazione di corporations multinazionali e, soprattutto, all'internazionalizzazione dei mercati finanziari. L'interdipendenza globale a livello economico ha contribuito a spingere grandi gruppi di individui dal Sud e dall'Est al Nord e all'Ovest. Infine, nel sistema politico, quello che ci interessa qui maggiormente, la globalizzazione implica un'intensificazione nell'espansione cross-nazionale di conflitti politici, la «transnazionalizzazione» delle relazioni politiche, il ruolo crescente di relazioni internazionali ed istituzioni politiche sovranazionali, e la sempre maggiore rilevanza dei problemi internazionali per le politiche nazionali.

In questo articolo, discuteremo le ripercussioni di queste trasformazioni per la mobilitazione dei movimenti sociali. Si può iniziare con l'osservare che $\mathrm{i}$ vari processi di globalizzazione sono riflessi in una diffusione della mobilitazione da parte dei movimenti sociali. Prima di tutto, come osserva Giddens $(1994,22)$, la globalizzazione non è solo «là fuori» ma anche «qua dentro»: essa trasforma la vita quotidiana e, di conseguenza, mobilita movimenti locali di resistenza che difendono le tradizioni locali contro l'intrusione di idee straniere e problemi globali. La recrudescenza del nazionalismo, i movimenti etnici, le mobilitazioni religiose così come dei fondamentalismi non sono niente altro che, nella definizione di Giddens, una «tradizione difesa in modo tradizionale» che deve essere vista sullo sfondo di questa intrusione. Allo stesso tempo, movimenti di solidarietà si mobilitano pro-attivamente su temi «distanti», cioè 
su temi che non sono immediatamente collegati al contesto nazionale ${ }^{1}$.

Nonostante l'importanza crescente dei fattori internazionali, transnazionali e sovranazionali, la sovranità dello stato-nazione continuerà, per il futuro prevedibile, a dominare tutte le altre forme di organizzazione della vita politica: gli stati-nazione sono ancora gli attori principali nelle relazioni internazionali, ed il contesto politico nazionale continua a filtrare in modo cruciale l'impatto del cambiamento internazionale sulle politiche nazionali. Istituzioni politiche nazionali e coalizioni politiche nazionali seguiteranno a plasmare i problemi sui quali le persone si mobilitano, i modi in cui esse si organizzano ed agiscono, e le conseguenze delle loro mobilitazioni. Ciononostante, come indicheremo nella prima sezione di questo articolo, il mutamento globale ha conseguenze significative per la mobilitazione all'interno dei contesti nazionali. Inoltre, la mobilitazione si estende ora oltre lo stato-nazione in modi che non erano stati previsti dai nostri modelli teorici. Tratteremo di queste profonde trasformazioni nella seconda sezione della nostra presentazione.

$\mathrm{Nel}$ corso della nostra discussione distingueremo due attori: a) i movimenti sociali e le loro constituencies, e $b$ ) i governi nazionali. Adattando al nostro contesto i concetti proposti da Knopf, possiamo individuare tre tipi di interazioni internazionali: interazioni transnazionali tra movimenti sociali di paesi diversi, interazioni transgovernative tra governi di paesi diversi, ed interazioni cross-livello tra un movimento sociale di un paese ed il governo di un altro paese ${ }^{2}$. Ad esse, si aggiungono le interazioni tra i movimenti sociali e i governi ad un livello sovranazionale.

\section{La mobilitazione nazionale in un mondo in via di globalizzazione}

Crescenti capacità di comunicazione cross-nazionale non creano automaticamente movimenti transnazionali: «la maggior

1 L'osservazione di Almond (1950) che i cittadini sono generalmente più indifferenti alla politica estera che alla politica interna potrebbe non riflettere più la realtà. Un'analisi particolareggiata di una serie di sondaggi su campioni rappresentativi di cittadini svizzeri dopo i voti su referenda federali durante gli anni ottanta e novanta ha indicato che, in generale, i cittadini tendono ad essere più interessati e meglio informati su problemi che riguardano le relazioni internazionali piuttosto che sui temi interni (Marquis 1997).

2 Knopf (1993) ha sviluppato questi concetti per l'analisi dei negoziati diplomatici, elaborando la concezione di Putnam di un gioco a due livelli (si veda più avanti). 
parte degli esempi che abbiamo attualmente non sono casi di movimenti globali con sedi a livello nazionale, ma di scambi politici tra attori alleati il cui contatto è stato facilitato dalla integrazione globale dell'economia e delle comunicazioni» (McAdam et al. 1996). Come già abbiamo accennato, la globalizzazione influenza il contesto nazionale nella misura in cui rende eventi che accadono a migliaia e migliaia di chilometri di distanza rilevanti per la mobilitazione locale. Per quanto riguarda i movimenti sociali, suggeriamo che la globalizzazione ha anche altre conseguenze sull'azione collettiva: in primo luogo, attraverso processi di diffusione, produce somiglianze cross-nazionali nella mobilitazione della protesta; in secondo luogo, mobilitazioni collettive intervengono nelle fasi di ratificazione dei negoziati internazionali; in terzo luogo, aumenta la rilevanza di opportunità e vincoli internazionali per i movimenti sociali nazionali.

Diffusione cross-nazionale e protesta. Un modo di pensare alla mobilitazione nazionale in un mondo globale è in termini di diffusione cross-nazionale. Gli studi sulla diffusione hanno una tradizione venerabile nelle scienze sociali, anche se non hanno mai assunto un ruolo centrale in nessuna di esse. Essi portano l'attenzione su di un punto importante, spesso ignorato dagli studiosi dei movimenti sociali: le campagne di mobilitazione politica non sono indipendenti l'una dall'altra, ma fanno parte di «un tessuto di relazioni spazio-temporali» (Goertz 1994,82 ). Osservare che la protesta politica è aggregata nel tempo e nello spazio non spiega automaticamente questa aggregazione. Infatti, «la sfida reale non è tanto dimostrare la mera esistenza della diffusione... quanto analizzare sistematicamente le condizioni nelle quali la diffusione è probabile ed i modi in cui essa si manifesta» (McAdam e Rucht 1993, 58).

Ci sono fondamentalmente due canali di diffusione: uno $d i$ retto ed uno indiretto. La diffusione diretta presuppone l'esistenza di un legame relazionale (formale o informale) diretto; la diffusione indiretta è basata sul trasferimento di informazione attraverso i mass media e, come osservano Strang e Meyer (1993), su collegamenti culturali. La globalizazione aumenta la densità di entrambi i tipi di canali. Idee e persone viaggiano facilmente nel «villaggio globale». Nel mondo globale, la facilità della comunicazione tra luoghi molto distanti crea e mantiene legami diretti sia di tipo interpersonale che di tipo interorganizzativo. $\mathrm{Ma}$ idee e modelli di azione collettiva possono diffon- 
dersi anche per vie indirette. Così, alcuni autori hanno suggerito che, nel caso della diffusione della protesta violenta in particolare, il meccanismo più probabile non è la comunicazione diretta, ma piuttosto un processo di apprendimento indiretto basato sulle informazione diffuse dai mass media (Archer e Gartner 1976; Spilerman 1976; Pitcher et al. 1978). Più in generale, ci aspetteremmo che la diffusione di idee e modelli dell'azione collettiva sia decisamente facilitata dalla «copertura» degli eventi di protesta da parte dei mezzi di comunicazione di massa. L'intensificazione cross-nazionale del dibattito pubblico e la corrispondente espansione del mercato delle idee costituiscono poi requisiti indispensabili per la diffusione delle campagne di protesta oltre i confini nazionali ${ }^{3}$. Esperti culturalmente legittimati - come scienziati, intellettuali, policy analysts e liberi professionisti - possono giocare una parte importante in questo processo (Strang e Meyer 1993). La questione cruciale rimane, tuttavia, capire in quali circostanze, quali tipi di idee sono recepite da quali attori.

Per quanto riguarda le condizioni che determinano la direzione della diffusione, troviamo nella letteratura due tipi di ipotesi: una ipotesi gerarchica e una ipotesi di prossimità (Collier e Messick 1975). Secondo l'ipotesi gerarchica, gli attori prendono a modello le unità più avanzate o più grandi all'interno di una certa popolazione. In questo quadro, un fenomeno quale la protesta politica tenderà ad espandersi con successo dalle unità più importanti a quelle meno avanzate o più piccole. Secondo l'ipotesi della prossimità, la vicinanza spaziale o la somiglianza culturale (cioè l'accettazione dal punto di vista culturale dell'appartenenza ad una certa categoria sociale) sono i fattori cruciali per la diffusione di un fenomeno nella misura in cui esse facilitano i legami tra unità sociali diverse $\mathrm{e}^{4}$.

3 Nell'ambito della policy analysis, Hall (1993) ha discusso la rilevanza per la diffusione di nuove idee del dibattito in corso nella società. Secondo Hall, mutamenti fondamentali nelle politiche pubbliche divengono più probabili quando lo sviluppo di un certo dibattito nella società coincide con un periodo elettorale. Possiamo aggiungere che quando da un dibattito nella società emergono forti mobilitazioni da parte di movimenti sociali, allora è probabile che queste mobilitazioni abbiano conseguenze sul piano elettorale.

4 In un articolo fondamentale sulla diffusione di innovazioni fra gli stati americani, Walker (1969) ha dimostrato che gli stati più grandi, più ricchi e più industrializzati adottano programmi nuovi più rapidamente di quelli più piccoli e meno sviluppati; inoltre, nelle diverse aree, vi sono stati-leader presi di solito a modello dai loro vicini. In un esempio più vicino al nostro argomento, Starr (1991) ha suggerito che effetti dimo- 
Relazioni gerarchiche o di prossimità possono esistere sia sul livello transgovernativo che su quello transnazionale, cioè sia tra governi che tra movimenti sociali. Ad esempio, a proposito della diffusione transgovernativa di idee e pratiche, McCarthy, McPhail e Crist (1999) hanno fatto uso di entrambe le ipotesi per spiegare l'espansione di quattro modelli diversi di controllo della protesta da uno stato all'altro (cfr. anche della Porta e Reiter 1998). Fattori gerarchici come la dipendenza coloniale e la coercizione sono, ad esempio, rilevanti nello spiegare l'espansione del modello irlandese nelle colonie britanniche. La prossimità geografica ha aiutato la diffusione del modello della polizia di Washington ai vari stati degli Stati Uniti e delle strategie di repressione della violenza degli booligans del calcio in Europa. Le autorità che sono responsabili per il controllo di polizia della protesta si osservano costantemente l'un l'altra alla ricerca di nuove idee. Come ha osservato Walker $(1969,889)$ nel corso della sua analisi dell'espansione di innovazioni fra stati americani, gli amministratori pubblici guardano ad altri stati dove problemi analoghi a quelli che essi devono affrontare sono stati affrontati con successo. Questo processo emulativo è una procedura euristica che permette loro di limitare i costi delle decisioni, ed influenza le capacità di adattamento delle autorità ai cambiamenti nelle forme di protesta.

Come quest'ultimo esempio indica, la diffusione transgovernativa porta a crescenti somiglianze nell'ambiente della protesta - per esempio nel modo in cui le autorità controllano le forme non-istituzionali di azione collettiva. Comunque, processi di diffusione coinvolgono i movimenti sociali anche in forme più dirette. In passato, la diffusione tra movimenti sociali era stata considerata come una forma inconscia di «contagio», una «reazione circolare» durante la quale ciascun individuo riproduce lo stimolo che riceve dal suo vicino (Blumer 1951). Dagli anni settanta, comunque, è stato riconosciuto che anche nel comportamento collettivo troviamo quegli stessi meccanismi di interazione interpretativa (mossi da identificazione ed imitazione, invece che da suggerimento e contagio) che caratterizzano le forme più «normali» di comportamento (Turner e Killian 1987). Come hanno notato McAdam e Rucht «i promotori della protesta non 
devono reinventarsi tutto in ciascun luogo ed in ciascun conflitto... Essi spesso trovano inspirazione altrove, nelle idee e nelle tattiche praticate da altri attivisti» $(1993,58)$.

Le idee relative a struttura organizzativa, strategie d'azione, o mete ideologiche «viaggiano» da una città all'altra, da un paese all'altro, dal centro alla periferia e, qualche volta, dalla periferia al centro. La ricerca sui movimenti sociali ha indicato che vari meccanismi di diffusione transnazionale sono all'opera contemporaneamente. Della Porta e Rucht (1995) hanno ad esempio suggerito che le somiglianze tra i movimenti della sinistra libertaria in Italia e nella Repubblica Federale Tedesca possono essere almeno parzialmente spiegate dalla diffusione cross-nazionale di temi, schemi di riferimento, repertori d'azione. In Svizzera, anche quando il contesto nazionale era molto dissimile da quello dei rispettivi paesi di riferimento, i gruppi di protesta nei cantoni di lingua francese hanno importato alcuni schemi di riferimento dai loro vicini in Francia, e lo stesso hanno fatto gli attivisti dei cantoni di lingua tedesca rispetto alla Germania, e quelli del Canton Ticino rispetto all'Italia (Wisler 1994). Nei primi anni sessanta, il movimento studentesco tedesco ha imparato da quello americano, quando gli studenti tedeschi hanno cominciato a trascorrere periodi di studio negli Stati Uniti. Più tardi, questi canali informali diretti hanno aiutato la creazione di una identificazione fra gli attivisti delle varie nazioni, e «una volta stabilitasi, questa identificazione ha permesso la diffusione attraverso una varietà di canali non-relazionali. Questi canali includevano la televisione, i gionnali, e le pubblicazioni sia di carattere scientifico che di carattere politico radicale» (McAdam e Rucht 1993, 71).

La diffusione non è comunque un processo di ricezione passiva di alcune idee elaborate altrove. Da un lato, essa comporta una selezione attiva da parte di chi «importa» le idee dall'esterno; dall'altro lato, essa spesso implica la promozione attiva da parte di chi trasmette una certa idea. Benford e Snow (1999) hanno infatti criticato il tacito assunto che la diffusione sia facilitata da somiglianze strutturali, suggerendo invece che le somiglianze sono simbolicamente costruite. Un processo di framing si svolge nel corso della diffusione cross-nazionale di riti, simboli e tattiche che sono adattati ad un ambiente nuovo. Inoltre, se le teorie sulla comunicazione della diffusione presuppongono l'esistenza di un emittente, un ricevente ed un messaggio trasmesso, nel caso di movimenti sociali è stata osservata 
una interazione pluridirezionale, con reciproca fertilizzazione, piuttosto che una chiara colonizzazione (Roggeband 1997, 3 e ss.; della Porta 1998).

Somiglianze nelle forme di mobilitazione tendono ad aumentare quando ondate internazionali di mobilitazione emergono in reazione ad eventi internazionali. L'incidente del reattore nucleare di Chernobyl, la guerra nel Vietnam o quella del Golfo, la decisione della Nato di installare nuovi missili a testata nucleare in vari paesi dell'Europa occidentale sono tutti eventi che hanno contribuito ad avviare ondate di protesta che hanno interessato contemporaneamente varie nazioni democratiche. In queste occasioni, gli scambi diretti ed indiretti tra attivisti dei movimenti nazionali erano particolarmente frequenti: riunioni internazionali sono state convocate, dimostrazioni di massa sono state organizzate negli stessi giorni in numerosi paesi, discorsi e documenti sono stati tradotti in varie lingue. Come risultato, specifici repertori di protesta o strutture organizzative si sono diffuse a vari movimenti nazionali: per esempio, durante la mobilitazione contro la installazione dei missili Cruise e Pershing II forme di azione diretta non violente sono state importate dal movimento della pace italiano, e ciò ha contribuito a moderare un conflitto politico che aveva assunto le forme più brutali del decennio precedente (della Porta 1995; della Porta 1996); e, ancora, sul modello del Greenham Common in Gran Bretagna, campi della pace sono stati organizzati anche in altri paesi europei e negli Stati Uniti (Rochon 1988). Anche eventi meno drammatici, come per esempio i congressi internazionali del Fondo Monetario Internazionale o della Banca Mondiale, hanno facilitato contatti fra attivisti provenienti da paesi diversi. Come ha osservato Rucht $(1993,81)$, «qualche volta questi contatti si intensificano e costituiscono la base per la costruzione di reticoli molto stretti, sebbene relativamente informali». Nel movimento ecologista, il network Ecoropa, che coinvolge attivisti da 15 paesi, o i Climate Action Networks sono esempi di questo genere.

Caratteristiche nazionali limitano comunque la capacità dei movimenti nazionali di «importare» protesta dall'esterno (Giugni 1995, 187 e ss.). In primo luogo, la disponibilità di pre-esistenti strutture di mobilitazione - sia di tipo interpersonale che di tipo organizzativo più formale - influenza lo sviluppo della protesta. Per esempio le differenze nella forza organizzativa dei movimenti nazionali aiutano a spiegare le reazioni diverse al disastro di Chernobyl in Francia, Paesi Bassi e Germania 
(Duyvendak e Koopmans 1995, 147 e ss.). In secondo luogo, avvenimenti internazionali assumono significati diversi in diversi contesti culturali (Goertz 1994, 34 e ss.) e non sempre gli schemi interpretativi adottati all'estero hanno una buona ricezione nel contesto nazionale. Per prendere ancora il caso della mobilitazione contro l'installazione dei missili della $\mathrm{Nato}$, in un'analisi comparata dei casi tedesco, britannico, italiano e francese, LeSaout (1995) ha osservato che i pacifisti francesi avevano molta più difficoltà nell'elaborare strategie simboliche capaci di neutralizzare l'accusa di essere strumenti, coscienti o meno, dell'Unione sovietica.

L'inserimento di un paese nel sistema internazionale degli stati-nazione e le opportunità politiche esistenti a livello nazionale costituiscono due ulteriori variabili rilevanti nel filtrare l'importazione di idee dall'estero. Per quanto riguarda la collocazione nel sistema di relazioni internazionali, la decisione di installare i missili a testata nucleare non poteva avere lo stesso impatto in paesi che non erano parte della Nato - quali la Francia o la Svizzera - e negli stati membri. Il caso dell'incidente al reattore nucleare a Chernobyl offre un esempio a proposito del ruolo delle opportunità politiche nazionali: la debole mobilitazione del movimento anti-nucleare in Francia è anche una conseguenza della reazione forte dello stato francese, che riuscì a convincere la popolazione «che le radiazioni si erano arrestate in qualche modo ai confini del paese, e che il discorso sulla pericolosità dei reattori sovietici non era rilevante a livello nazionale data la tecnologia superiore degli impianti nucleari francesi» (Duyvendak e Koopmans 1995, 163). Nel caso della Guerra del Golfo, se i tempi della mobilitazione furono simili in vari paesi, le capacità della mobilitazione contro la guerra si dimostrarono estremamente diverse in Germania, Francia ed Olan$\mathrm{da}$, soprattutto a causa della diversa struttura di alleanze disponibile per i movimenti, così come della risonanza del discorso pacifista nella cultura nazionale (Koopmans 1999).

Movimenti sociali in un gioco a due livelli. La globalizzazione influenza i movimenti sociali a livello nazionale anche aumentando il ruolo delle mobilitazioni nazionali sui negoziati internazionali. Putnam ha introdotto il concetto di gioco a due livelli come metafora per riferirsi alle interazioni nazionali-internazionali nei negoziati internazionali: 
La politica di molti negoziati internazionali può essere utilmente concepita come un gioco a due livelli. Al livello nazionale, gruppi locali perseguono $i$ loro interessi facendo pressione sul governo perché adotti politiche ad essi favorevoli, mentre i politici cercano di ottenere potere costruendo coalizioni fra vari gruppi. Al livello internazionale, i governi nazionali cercano di massimizzare la loro abilità di soddisfare le pressioni interne, minimizzando le conseguenze negative all'esterno. Né l'uno né l'altro dei due giochi può essere ignorato dai decision-makers, fino a che i loro paesi rimangono interdipendenti e, tuttavia, sovrani $(1988,434)$.

Per tenere conto di questi due livelli, Putnam propone di scomporre i negoziati internazionali in due fasi - una fase di negoziazione, durante la quale il negoziato viene condotto dai negoziatori, portando ad un accordo provvisorio; ed una fase di ratificazione, durante la quale varie discussioni si svolgono separatamente, all'interno dei diversi paesi, sulla opportunità di ratificare l'accordo. Il risultato della fase del negoziato dipende, naturalmente, dalle strategie ed abilità del negoziatore. Il risultato della fase di ratifica dipenderà, invece, dalla struttura istituzionale e da preferenze e coalizioni esistenti sul livello nazionale.

$\grave{\mathrm{E}}$ ovvio che in un mondo sempre più globale i negoziati internazionali divengono sempre più importanti ed il crescente interesse del pubblico per le relazioni internazionali aumenta la rilevanza della fase di ratificazione nel gioco a due livelli che abbiamo appena descritto. Un caso senz'altro pertinente è la ratifica delle varie tappe di espansione e rafforzamento dell'Unione europea, quando referenda nazionali sono stati organizzati in vari paesi europei (Luthardt 1992). È concepibile che, in queste circostanze, movimenti sociali nazionali divengano giocatori sempre più importanti nei negoziati internazionali. Le implicazioni di questo sviluppo sono ambigue: l'esistenza di movimenti nazionali contrari ad un trattato internazionale può aumentare le capacità di negoziato del negoziatore di quel paese, almeno all'inizio del processo. Essa comunque può, allo stesso tempo, ridurre le occasioni di cooperazione internazionale. La Svizzera è una buona illustrazione di questo punto: il fatto che i trattati internazionali importanti devono essere sottoposti a un voto popolare il cui risultato è vincolante per $\mathrm{i}$ negoziatori ha fino ad oggi impedito l'ingresso del paese nella Unione europea.

Vincoli ed opportunità internazionali. Un terzo effetto della globalizzazione è la crescente rilevanza dell'ambiente internazionale per i movimenti sociali nazionali. Vincoli ed opportunità in- 
ternazionali possono intervenire sia nelle relazioni transgovernative, che in quelle transnazionali e cross-livello (si veda sopra).

Guardiamo prima ai vincoli internazionali. Un approccio interessante ai vincoli internazionali alla mobilitazione politica in un contesto nazionale è offerto da Goertz (1994) con il così detto «modello della barriera». Benché Goertz focalizzi l'attenzione sul livello transgovernativo, il suo modello può essere utile anche ad analizzare lo sviluppo di movimenti sociali in stati diversi che devono fronteggiare vincoli simili a causa delle pressioni transgovernative esercitate sul loro governo da un potere straniero - una superpotenza, una potenza coloniale o un attore dominante a livello regionale o settoriale. Questa pressione potrebbe spingere $\mathrm{i}$ governi nazionali a reprimere la mobilitazione aperta e/o bloccare la realizzazione di alcune riforme. Secondo questo modello, i movimenti sociali nei diversi paesi sottomessi a questa relazione di potere transgovernativo guardano all'autorità che mantiene la barriera e periodicamente mettono alla prova la sua forza.

Un cambiamento nel contesto internazionale può indebolire questi vincoli, e addirittura creare opportunità all'azione di protesta. Se la barriera perde forza nel corso del tempo, una volta che la conoscenza di tali cambiamenti si diffonde fra i movimenti, essi si possono mobilitare in alcuni paesi, aumentando così la pressione sulla barriera, la quale si può rompere in uno o più punti. Il successo dei movimenti che si mobilitano per primi ${ }^{5}$ ha due effetti sul livello transnazionale: movimenti simili in altri paesi sono spinti a mobilitarsi e, allo stesso tempo, imparano dai movimenti che hanno avuto successo le strategie utili a realizzare i loro obiettivi. Questo duplice effetto faciliterà il crollo della barriera e l'emergere di una ondata di movimenti nei diversi paesi che erano sottoposti al vincolo esterno. Goertz (1994, 137 170) applica questo modello agli sviluppi dal 1945 fino a 1989 nell'Est Europa, dove la barriera è stata costituita evidentemente da Mosca. Anche per quanto riguarda i movimenti sociali in Europa Orientale, la liberalizzazione del regime politico nell'Unione Sovietica ha rimosso un potente ostacolo alle mobilitazioni di massa che si sono infatti sviluppate in tutte le nazioni dell'Est europeo, benché con forza e conseguenze diverse ${ }^{6}$. (1996).

5 Per una distinzione tra movimenti initiatori e derivati si rinvia a McAdam

6 Per un'analisi comparata della transizione nell'Europa dell'Est e del ruolo giocatovi dai movimenti sociali, cfr. Chilton (1995). 
Opportunità internazionali per la protesta possono operare in modo ampiamente analogo. In questo caso la barriera si ha a livello nazionale mentre un attore esterno appoggia i movimenti sociali dei paesi sottoposti a quella barriera. Il sostegno esterno, sia da parte di governi stranieri che da parte di movimenti sociali in paesi stranieri, permette ad un movimento sociale di un dato paese di fare pressione laddove il contesto è meno ostile che a livello nazionale. Il suo successo faciliterà allora il successo di movimenti sociali in altri paesi che hanno di fronte vincoli simili nel loro contesto nazionale. La strategia di Che Guevara e del governo cubano negli anni sessanta e nei primi anni settanta così come l'appoggio per movimenti di liberazione in America Centrale (Salvador, Nicaragua o Guatemala) o nell'Africa Meridionale (Angola, Mozambico, Sud Africa) da parte di organizzazioni del movimento di solidarietà del Nord del mondo hanno seguito un approccio simile.

Rispetto ai modelli della diffusione, i modelli dei vincoli e delle opportunità internazionali hanno il vantaggio di collocare l'interazione tra autorità e sfidanti al centro dell'attenzione. Essi sono, perciò, compatibili con le più recenti teorie che, sia nella letteratura sui movimenti sociali che in quella sulla democratizzazione, sottolineano il ruolo dell'attore all'interno di un contesto strutturale (cfr. Pagnucco 1995; Stark e Bruszt 1990). La capacità esplicativa appare ancora aumentata da una combinazione dei modelli dei vincoli e delle opportunità. Così, il modello delle opportunità può essere usato per spiegare la neutralizzazione dei vincoli esterni. Nel caso dell'Europa Orientale, per esempio, i mezzi di comunicazione di massa occidentali hanno probabilmente costituito una importante forza di pressione che ha contribuito ad indebolire la barriera costituita dal potere dell'Unione Sovietica (Chilton 1995).

Riassumendo, in un mondo interdipendente è sempre più facile per i movimenti sociali di un certo paese ottenere l'appoggio di movimenti sociali di un altro paese, più ricco di risorse di partecipazione, o di ottenere l'appoggio del governo di un altro paese per aumentare la pressione sul proprio. In questo caso l'obiettivo della protesta è ancora il governo nazionale, ma il pubblico di riferimento sono i movimenti sociali o le autorità in un altro paese. Si hanno così mobilitazioni transnazionali, tra movimenti sociali di vari paesi, e/o mobilitazioni cross-livello, che coinvolgono un movimento di un paese e un governo di un altro paese. Un esempio è il movimento Anti-Apartheid fuori 
dal Sud Africa. In questo caso, il movimento sudafricano ha stimolato una mobilitazione in molti paesi occidentali dove movimenti di solidarietà hanno fatto pressione sui loro governi per un boicottaggio dei prodotti sudafricani, in modo da costringere il governo sudafricano a rinunciare alla politica di segregazione razziale.

Quando questo tipo di mobilitazioni avrà più probabilità di successo? Tre tipi di condizioni sembrano particolarmente rilevanti: il tipo di relazioni esistenti tra il governo bersaglio della protesta e quelli chiamati ad intervenire; la struttura delle opportunità politiche nel paese bersaglio della protesta; la struttura delle opportunità politiche nei paesi chiamati ad intervenire. Le relazioni gerarchiche tra governi-bersaglio e governi chiamati ad intervenire - quali ad esempio precedenti vincoli di natura coloniale - o la prossimità spaziale o culturale tra due paesi facilitano l'esercizio di pressione a livello transgovernativo. Così, la capacità di incidenza del movimento per i diritti civili nel Sud Africa è stata aumentata dalla esistenza di legami con i paesi che avevano in passato colonizzato il paese e da una lingua comune. Inoltre, le pressioni a livello transgovernativo sembrano avere possibilità di successo quanto più il paese del governo bersaglio dell'intervento è integrato nella comunità internazionale e quanto maggiore è il suo desiderio di essere considerato un membro rispettabile della comunità internazionale. Come ha rilevato Sikkink (1993) comunque, la pressione transgovernativa è meno efficace nel caso di paesi che hanno una certa rilevanza per la sicurezza nazionale delle superpotenze.

Circostanze interne fungono naturalmente da filtri delle pressioni che vengono da altri paesi. Risse-Kappen ha osservato che una questione centrale per l'analisi delle relazioni transnazionali riguarda i loro effetti differenziali su contesti nazionali: «Come può essere spiegato, per esempio, che l'espansione di valori di democrazia e diritti umani verso la fine di questo secolo, promossa da varie organizzazioni internazionali non-governative ed alleanze transnazionali, ha riguardato alcuni paesi più che altri - la ex-Unione Sovietica più della Cina, la ex-Cecoslovacchia più che la Romania, e la Corea del Sud più che la Corea del Nord?» $(1995,4)$. Oltre che il grado di istituzionalizzazione del sistema di relazioni internazionali attorno ad un certo tema, la risposta a questa domanda deve prendere in considerazione i sistemi organizzativi e normativi dei singoli stati (cfr. anche Chilton 1995; Princen 1995). 
Per quanto riguarda le opportunità politiche nazionali nel paese chiamato ad esercitare pressione, il movimento sociale nel paese sul quale si vuole esercitare pressione deve adattare il suo repertorio d'azione alle norme diffuse nel paese di cui chiede l'appoggio - l'accento sulla protesta non-violenta risponde, almeno in parte, all'obiettivo di non alienarsi le simpatie occidentali. In analogia al noto modello di Lipsky sull'uso della protesta come risorsa politica (Lipsky 1965), si può osservare comunque che uno dei problemi principali per i movimenti sociali dei paesi-bersaglio è che essi devono fare affidamento sulla opinione pubblica internazionale che può essere facilmente soddisfatta da risposte puramente simboliche da parte dei governi.

\section{Mobilitazione oltre lo stato-nazione}

Se lo stato-nazione rimarrà il contesto politico più importante per la mobilitazione dei movimenti sociali per ancora molto tempo, ci sono comunque alcuni segnali che indicano che esso non ne è più il teatro esclusivo. I sistemi politici hanno sempre avuto una stratificazione complessa a più livelli, con opportunità regionali e locali a completare quelle esistenti a livello nazionale. Con lo sviluppo di istituzioni politiche intergovernative, come l'Unione europea e le Nazioni unite, un livello sovranazionale si è aggiunto ai precedenti aprendo nuove possibilità per i movimenti sociali.

Seguendo, più o meno esplicitamente, gli approcci di relazioni internazionali che enfatizzano la crisi della nazione e la moltiplicazione di centri decisionali (ad esempio, Rosenau 1990), diversi studiosi dei movimenti per i diritti umani o dei movimenti ecologisti hanno cominciato ad interessarsi del potere crescente delle International Governmental Organizations (IGOs), e del moltiplicarsi delle International Non-Governmental Organizations (INGOs). È stato così coniato il concetto di organizzazioni di movimento sociale transnazionali (TSMOs, nell'acronimo inglese) (Smith et al. 1994; Sikkink 1993; Chatfield et al. 1997). Studiosi di relazioni internazionali hanno inoltre parlato di una «politica civica mondiale» per riferirsi a «quella componente della vita associativa che esiste sopra l'individuo e sotto lo stato, ma anche oltre i confini nazionali» (Wapner 1995, 313; si veda anche Wapner 1996). Insieme agli stati, anche vari tipi di organizzazioni sono considerate come attori 
importanti di una multi-track diplomacy ${ }^{7}$, mentre approcci bottom-up hanno sottolineato il ruolo della partecipazione locale e dei movimenti di base nella politica internazionale (Princen 1994, 33). A dimostrare la rilevanza di questo livello sovranazionale, basti ricordare che, da 1909 al 1988, il numero delle IGOs è salito da 37 a 309 e quello delle INGOs da 176 a 4.518 (Princen e Finger 1994, 1; cfr. anche McGrew 1992, 7-12). In che misura le IGOs siano divenute bersagli o arene per lo sviluppo di attività dei movimenti sociali è, comunque, una questione ancora aperta alla quale cercheremo di fornire nelle pagine che seguono qualche elemento di risposta, per poi guardare alle forme che la mobilitazione assume a livello transnazionale.

Problemi sovranazionali, obiettivi nazionali. Possiamo cominciare con l'osservare che la costruzione di istituzioni sovranazionali costituisce oggi l'oggetto di vivaci contestazioni. Riguardo all'Unione europea possiamo notare, insieme a Hooghe e Marks (1995), che «l'integrazione europea non opera più sotto la copertura tecnocratica ma è determinata invece da un continuo ed esplicito conflitto politico fra coalizioni di governi, attori sovranazionali, ed interessi nazionali». Fra i rappresentanti di interessi nazionali troviamo anche i movimenti sociali. Da un lato ci sono movimenti nazionalistici, che mettono in discussione la praticabilità del processo di europeizzazione. Dall'altro lato, ci sono movimenti che mobilitano risorse in sostegno dell'integrazione europea o movimenti - come Eurotopia - che propugnano una democratizzazione delle istituzioni europee. In altre parole, il processo di creazione di un governo sovranazionale fornisce uno stimolo cruciale per la mobilitazione di movimenti sociali nell'Europa contemporanea. Le conseguenze di questi conflitti sono ancora incerte. Secondo un attento osservatore (Schmitter 1993/94; Schmitter, in stampa), il processo non sfocerà nella fine dello stato-nazione, ma piuttosto nella costruzione di una nuova forma di governo a più strati - che include strutture variabili a livello funzionale e territoriale. Secondo la sua nozione di «condominio», che riprende l'idea di una Europa a «geometria variabile» o di una Europa a «più velocità», ci saranno probabilmente non una, ma molte Europe: sottoinsiemi

7 Come, per esempio, i gruppi di donne attivi sul tema della pace nel conflitto israeliano-palestinese (Sharoni 1995). 
multipli di stati membri che agiscono autonomamente per risolvere problemi comuni e produrre beni pubblici. Tale esito è reso ancora più probabile dal fatto che la lotta politica per l'integrazione europea tende ad avere conseguenze specifiche nei diversi paesi, a seconda del loro assetto istituzionale e della configurazione delle coalizioni al potere. Per i movimenti sociali, una «Europa condominio» implica opportunità molto diverse a seconda del tipo di problema oggetto della mobilitazione ${ }^{8}$.

Nella sua struttura presente l'Unione europea è principalmente un mercato comune ed i governi nazionali costituiscono gli intermediari privilegiati tra le politiche nazionali e il negoziato europeo (Hooghe e Marks 1995). I dirigenti nazionali fissano l'agenda e negoziano le decisioni più rilevanti, e solo più tardi riportano le decisioni agli attori nazionali che le possono alterare solo con grande sforzo e a costi considerevoli. Nonostante i progressi fatti verso la creazione di una cittadinanza europea e la maggiore rilevanza dei canali democratici al livello sovranazionale, l'accesso alle decisioni rimane ancora molto limitato. In questa situazione, i movimenti sociali, così come gli altri attori non-governativi che rappresentano interessi nazionali, difficilmente ottengono accesso al livello europeo di governo?

In questo senso la mancanza, o debolezza, di accountability democratica della maggior parte delle istituzioni sovranazionali le mette al riparo dalle pressioni dal basso. Come Eisinger (1973) aveva indicato nella sua ricerca sui movimenti sociali nelle città americane, le opportunità politiche per la protesta sono minori dove istituzioni governative non sono elette dai cittadini (per esempio, nei governi locali «riformati» dove il sindaco è un burocrate); viceversa, esse aumentano quando l'autorità istituzionale è responsabile di fronte all'elettorato. Parallelamente, possiamo affermare che le opportunità istituzionali a livello internazionale sono formalmente limitate quando c'è un livello molto basso di controllo (come nell'Ue), o nessun controllo (come nell'Onu) da parte del cittadino-elettore.

Inoltre, in un sistema politico multi-strato che si sviluppa a velocità diverse in direzioni multiple, quale è l'Unione europea,

8 In un'analisi della mobilitazione regionale nell'Ue, Marks et al. (1996) hanno osservato una correlazione positiva tra rappresentanza cross-nazionale e grado di sovrapposizione tra le competenze dei governi subnazionali e quelle dell'Ue.

9 Per esempio, il livello di mobilitazione dei gruppi ambientalisti rispetto alla Unione europea è molto basso (Rucht 1997). 
la responsabilità è difficile da localizzare. Infatti, decisioni che risultano da un negoziato intergovernativo al livello europeo sono poi implementate in maniera decentrata e differenziata nei diversi paesi. Per i cittadini spesso è difficile identificare le fonti della loro eventuale insoddisfazione e i possibili referenti delle loro domande di riforme ${ }^{10}$.

Data la relativa inaccessibilità del livello sovranazionale ed il ruolo cruciale che i governi nazionali ancora giocano nel processo politico al livello europeo, è probabile che i bersagli della mobilitazione dei movimenti sociali continuino ad essere i governi nazionali. Non è quindi una sorpresa che Imig e Tarrow (1999) (cfr. anche Tarrow 1995) abbiano trovato un numero limitato (sebbene in aumento) di eventi di protesta direttamente indirizzati al livello europeo e ipotizzato che la crescente rilevanza delle politiche a livello europeo si rifletterà non tanto in proteste contro l'Unione europea quanto piuttosto in rivendicazioni rivolte ai governi nazionali.

Non bisogna comunque concludere che l'aggiungersi di un livello sovranazionale di governo non abbia nessuna rilevanza per la mobilitazione di movimenti sociali. Prima di tutto, la chiusura formale di queste istituzioni può essere bilanciata da una strategia prevalente (Kriesi 1991) orientata al negoziato. Come hanno osservato Marks e McAdam (1999), organizzazioni di movimento che si adattano alle loro regole del gioco possono ottenere un accesso routinizzato, sebbene prevalentemente informale, alle organizzazioni sovranazionali. Questo sembra essere il caso, per esempio, delle TSMOs attive sul diritto delle popolazioni indigene che sono riuscite ad influenzare in modo rilevante le decisioni dell'Onu attraverso un'azione di sensibilizzazione dell'opinione pubblica, ma anche di lobbying dei rappresentanti delle diplomazie di vari paesi (Passy 1999).

Si può aggiungere che il grado di apertura formale ed informale alle NGOs varia da una IGO all'altra. Così, nel confronto con l'Ue, l'Onu è più aperto alle NGOs, almeno al livello della consultazione. Infatti, l'Art. 71 della Carta delle Nazioni unite consente alle organizzazioni non governative di fare domanda per ottenere lo status consultivo presso il Consiglio economico

10 Ad esempio, Klandermans et al. (1999) hanno illustrato la confusione dei cittadini nel fronteggiare questo nuovo tipo di Leviatano nell'area della politica agricola un'area che è stata al centro dell'attenzione fin dai primi passi della integrazione europea. 
e sociale (Ecosoc). Alle TSMOs con lo status consultivo viene quindi permesso di inviare un osservatore a quelle sessioni dell'Ecosoc che sono rilevanti ai loro fini e di ricevere la documentazione relativa (Smith 1995, 199) ${ }^{11}$.

Arene sovranazionali. Anche se non sono spesso bersaglio della protesta, organizzazioni sovranazionali - ed un nuovo pubblico di riferimento ad esse collegato: il pubblico europeo, o il pubblico globale - rappresentano sempre più spesso arene per l'articolazione di rivendicazioni collettive. Intorno a temi quali i diritti umani o l'uguaglianza fra i sessi, alleanze (o policy networks) si costruiscono spesso tra organizzazioni di movimento sociale ed istituzioni governative internazionali (Chatfield et al. 1997). Le organizzazioni transnazionali di movimento sociale possono offrire alle IGOs due risorse delle quali queste ultime sono spesso carenti: informazione e legittimazione. Dal canto loro, le IGOs possono offrire alle TSMOs risorse sia materiali che simboliche ${ }^{12}$. Conferenze patrocinate dalle Nazioni unite come la conferenza sull'ambiente e lo sviluppo nel 1992, la conferenza sui diritti umani nel 1993, o le quattro conferenze sul tema delle donne nel 1975, 1980, 1985 e 1995 - hanno costituito importanti arene per l'intervento di organizzazioni transnazionali di movimento sociale. Non a caso, ben 1.500 sono le NGOs accreditate alle Nazioni unite.

L'importanza di questa arena sovranazionale varia per i diversi movimenti nazionali. È soprattutto quando le istituzioni politiche nazionali sono più chiuse che gli attivisti dei movimenti sociali possono cercare l'appoggio di IGOs per fare pressione sul loro governo. Opportunità politiche transnazionali

11 Questa apertura è il risultato dall'azione di 1.200 rappresentanti di NGOs che ha fatto pressioni sui loro rappresentanti nazionali al momento della stesura della Carta delle Nazioni unite (Leary 1979).

12 Per esempio nel 1992, il Directorate-General for Environment, Nuclear Safety and Civil Protection ha speso di 6,5 milioni di ecu per il finanziamento di gruppi non governativi attivi su temi ambientali (Rucht 1993, 87). Attraverso l'Echo (European Commission Humanitarian Office), l'Ue è uno sponsor importante delle NGOs che operano sul tema dei diritti umani (Eberwein 1997). Nei paesi in via di sviluppo, grazie alle loro competenze tecniche e ai loro contatti «sul campo», NGOs locali sono divenuti attori importanti nella distribuzione dei finanziamenti provenienti dai paesi più sviluppati (per un totale di 1,1 miliardi di dollari americani nel 1985) (Wapner 1995, 334). Alla fine degli anni ottanta, il $10-15 \%$ dei fondi per l'assistenza allo sviluppo dei paesi dell'Ocse era incanalato attraverso NGOs con sedi nei paesi più sviluppati (Princen 1994, 34). 
possono essere, per esempio, molto importanti durante i processi di democratizzazione, quando l'adozione di diritti umani come principale schema di riferimento per l'opposizione a regimi autoritari può aiutare lo sviluppo di alleanze internazionali. Organizzazioni internazionali, come l'Unione europea, la Organization of the American States e l'Association of Southeast Asian Nations, hanno giocato un ruolo importante in alcuni dei processi recenti di democratizzazione: «i vincoli politici che esse hanno imposto, insieme con il sostegno materiale mobilitato o le sanzioni economiche collettive applicate non sempre sono state sufficienti a prevenire ritorni indietro all'autoritarismo e alla violenza... ma hanno condotto a successi importanti» (Karl e Schmitter 1994, 58). Negli anni ottanta e novanta, una «società civile transnazionale» ha contribuito alla sopravvivenza delle nuove democrazie attraverso vari tipi di intervento: c'è stata infatti una «costante crescita di organizzazioni internazionali nongovernative che offrono servizi per le nuove democrazie, monitorando l'andamento delle elezioni e i diritti umani, e anche mobilitando sostegno quando esse sono minacciate da colpi di stato militari, brogli elettorali, o azioni arbitrarie del governo» (Karl e Schmitter 1994, 57-58). Una ricerca sul processo di democratizzazione in Argentina ha ad esempio illustrato l'impatto che arene internazionali e una opinione pubblica sovranazionale possono avere sulle democrazie in via di sviluppo ${ }^{13}$.

Strutture transnazionali di mobilitazione. La disponibilità di arene sovranazionali può avere conseguenze importanti sulla struttura dei movimenti sociali. Così come i movimenti sociali sono divenuti attori nazionali con l'affermarsi dello stato-nazione (Tilly 1984), lo sviluppo di arene politiche sovranazionali ha prodotto attori collettivi transnazionali. Infatti, le organizzazioni transnazionali dei movimenti sociali - quali Greenpeace, Amnesty International, Friends of the Earth o le Peace Brigades International - si sono moltiplicate. Parte del gruppo molto più ampio delle così dette organizzazioni nongovernative, le

13 Analizzando la mobilitazione per i diritti umani in Argentina, Brysk ha concluso che «la pressione internazionale è efficace - almeno in alcune aree» $(1993,261)$. La battaglia degli attivisti argentini per i diritti umani ha trovato alleati ed una arena per esprimersi in un regime internazionale per i diritti umani - comprendente organizzazioni quali l'Human Rights Commission delle Nazioni unite, creato nel 1976, e l'Human Rights Commission dell'Oas, oltre a numerose TSMOs. 
TSMOs mobilitano primariamente risorse che provengono dalla loro base di riferimento, e solo in modo più limitato dal sostegno di governi o di altre istituzioni. Specialmente negli anni ottanta, TSMOs e INGOs sono cresciute in termini di numero di unità organizzative, numero di membri e quantità di risorse materiali per esse disponibili.

Il loro sviluppo è stato particolarmente intenso in quelle aree dove maggiore è il ruolo delle IGOs: in particolare, ambiente, diritti umani e diritti delle donne. Le organizzazioni transnazionali nel campo della ecologia «si sono moltiplicate esponenzialmente dagli anni settanta in poi, con un bilancio eguale, se non maggiore, alla spesa annuale del programma per l'ambiente delle Nazioni unite» (Wapner 1995, 315). Grazie ad un ammontare di risorse considerevole e crescente, TSMOs sui temi ambientali hanno cercato di influenzare le decisioni delle IGOs in questo campo: oltre ad organizzare azioni di protesta, esse fanno pressione su istituzioni nazionali ed internazionali, arrivando a pagare le spese di viaggio ai delegati dei paesi più poveri in modo da permettere loro di frequentare riunioni internazionali importanti o aiutandoli con servizi di traduzione simultanea durante le riunioni informali che accompagnano le conferenze internazionali, o attraverso il così detto debt-for-nature (cioè l'acquisto del debito di un paese povero da parte di una banca o di un istituto di credito in cambio di politiche di protezione dell'ambiente) (Schaefer Caniglia 1995, 11 e ss.) ${ }^{14}$.

Scrivendo sul movimento per i diritti umani, Smith ha osservato che «una struttura transnazionale delle opportunità politiche influenza le capacità di mobilitazione dei movimenti così come le loro alternative strategiche» $(1995,187)$. INGOs, come Amnesty International, la International Commission of Jurists, la International Ligue for Human Rights, la International Federation for Human Rights, la International Association of Democratic Lawyers, hanno giocato un ruolo centrale nella lotta contro le violazioni del diritti umani ${ }^{15}$. Sempre più spesso, INGOs

14 Nel 1993, 22.000 rappresentanti di 9.000 NGOs hanno partecipato alla Conferenza delle Nazioni unite sull'ambiente e lo sviluppo a Rio. NGOs hanno giocato anche un ruolo importante nella conferenza delle Nazioni unite sul clima nel 1995 a Berlino (Walk e Brünnengräber 1996; Janett 1996). Sempre più spesso, IGOs accordano ad NGOs attive su temi ambientali lo status di osservatori (Princen e Finger 1994, 5 e ss.).

15 Amnesty International ha giocato un ruolo importantissimo per lo sviluppo di standard internazionali contro la tortura, ricevendo il premio Nobel per la Pace nel 1977 (Leary 1979). 
attive sul tema dei diritti umani intervengono in modo coordinato rispetto alle IGOs nelle emergenze internazionali - come, in particolare, nella guerra nella ex-Jugoslavia (Baglioni 1998). Per quanto riguarda il movimento delle donne, basti osservare che il numero dei delegati delle NGOs alle conferenze delle Nazioni unite sulle Donne è cresciuto da 5.000 a Città del Messico nel 1975 a 30.000 a Pechino nel 1995 (Holthaus 1996, 61). Sembra quindi che tanto più importante è il contesto internazionale, tanto più le organizzazioni dei movimenti sociali tendono ad aumentare la loro presenza transnazionale.

Le interazioni con le organizzazioni governative internazionali influenzano le caratteristiche dei movimenti sociali. Come è stato notato (Smith et al. 1994, 126), perché i movimenti sociali si possano impegnare con successo nelle arene sovranazionali, essi devono sviluppare una base di risorse transnazionale e strategie globali che probabilmente variano in modo significativo rispetto a quelle usate nelle arene nazionali. In primo luogo, queste arene offrono infatti a rappresentanti di movimenti di diverse regioni del mondo l'opportunità per incontrarsi, tessere reti organizzative, coordinare le loro attività, costruire un discorso globale (Passy 1999; Smith 1999) ${ }^{16}$. In altre parole, l'esistenza di arene sovranazionali rafforza la dimensione transnazionale dei movimenti. Inoltre, la cooperazione transnazionale può condurre ad una centralizzazione e strutturazione delle TSMOs. Per i gruppi ecologisti, «qualunque siano le loro dimensioni, strutture e tematiche, sembra esserci un andamento generale $a$ ) verso una maggiore collaborazione (sebbene non sempre definita in modo formale) e $b$ ) verso una maggiore specializzazione e professionalizzazione, che poi sfocia in una implicita divisione del lavoro» (Rucht 1993,87$)^{17}$. Inoltre, per potere ricevere sostegno economico dalle IGOs, le TSMOs devono adattare la loro struttura organizzativa, accettando controlli esterni, adottando codici di condotta, e così via ${ }^{18}$.

Gli effetti delle interazioni con una sfera sovranazionale

16 Ad esempio, sui temi dell'ecologia, varie organizzazioni di movimento sociale hanno creato una rete di comunicazione transnazionale, usando siti internet (quali EcoLink o PeaceNet) per mobilitare un'opinione pubblica internazionale (Schaefer Caniglia 1995, 13).

17 Cfr. Marx Ferree e Gamson (1999) per osservazioni simili a proposito del movimento del donne.

18 Per esempio, si veda Eberwein (1997) sugli effetti della collaborazione con l'Echo dell'Ue sulle INGOs in essa coinvolte. 
sembrano comunque diversi per i diversi movimenti sociali ed organizzazioni di movimento sociale. Come hanno sottolineato Marks e McAdam (1999), la probabilità che movimenti sociali facciano il salto a livello sovranazionale dipende non solo dal potere delle organizzazioni intergovernative sui problemi specifici sui quali un movimento si mobilita, ma anche dalla cultura degli attori non istituzionali. Per esempio, dato il suo tradizionale radicamento nello stato-nazione, il movimento operaio mostra particolari difficoltà ad adattarsi allo sviluppo di una struttura istituzionale europea (cfr. anche Traxler e Schmitter 1995, 207).

Su diversi problemi, le arene sovranazionali costituiscono un luogo di incontro per svariati tipi di organizzazioni che spesso si alleano a sostegno delle stesse mete condivise da specifici movimenti sociali. Per esempio nel caso dei diritti umani, Sikkink $(1993,415)$ ha osservato la presenza di principled issue networks, definiti come «un insieme di organizzazioni legate l'una all'altra da valori condivisi e da un intenso scambio di informazioni e servizi, che lavora a livello internazionale su un certo tema». Composti da TSMOs, altre NGOs, organizzazioni intergovernative internazionali e fondazioni private, questi reticoli identificano, documentano e denunciano violazioni dei diritti umani in un dato paese e fanno pressione perché i governi di altri paesi intervengano contro chi viola i diritti umani ${ }^{19}$. Un esempio discusso da Sikkink è la persecuzione dell'editore ebreo Jacobo Timerman in Argentina, contro la quale si mobilitarono organizzazioni per i diritti umani, membri della comunità ebraica degli Stati Uniti e giornali americani.

Ancora un altro tipo di strutture di mobilitazione, che operano sul livello transnazionale, sono le così dette comunità epistemiche, definite come «una rete di professionisti con competenze riconosciute in settori specifici ed autorevolezza in campi di conoscenza rilevanti per una certa policy» (Haas 1992). Sul tema della messa fuori legge del cloro-fluoro-carbonio, Haas ha identificato una comunità transnazionale, composta dal Programma per l'ambiente delle Nazioni unite, dalla Agenzia per la protezione ambientale degli Stati Uniti, dall'Ufficio del Dipartimento di Stato degli Stati Uniti sugli Ocean and International

19 In modo simile, Princen (1995) ha usato il termine di «coalizioni ambientaliste transnazionali» per definire la rete di gruppi che si sono mobilitati sul problema del traffico illegale dell'avorio. 
Environmental and Scientific Affairs (Oes) e dagli scienziati dell'atmosfera nella comunità scientifica internazionale. Questa «comunità epistemica» ha giocato un ruolo di primo piano attraverso la raccolta di informazioni, la costruzione di un ampio consenso attorno a queste informazioni, la loro diffusione a governi e grandi gruppi industriali, e l'aiuto ai decision-makers nella formulazione di politiche pubbliche relative alla produzione e al consumo di CFC. I membri di questa comunità epistemica hanno avuto così successo nel convincere della necessità di forti controlli in tema di CFC due attori decisivi: il governo americano e la DuPont, azienda leader nella produzione di CFC a livello mondiale.

Nel caso del bando del cloro-fluoro-carbonio così come nell'intervento sulle violazioni dei diritti umani i repertori d'azione sono stati sorprendentemente simili: gli attori coinvolti hanno raccolto informazioni e documentato le loro posizioni in modo da essere persuasivi. Infatti, l'uso di arene sovranazionali coinvolge tipicamente i partecipanti in un gioco in cui un valore fondamentale ha la raccolta di informazioni, la elaborazione di documenti, la pressione su chi formula risoluzioni finali. Il movimento dei popoli indigeni si è mosso in maniera simile all'interno dell'Onu (Passy 1999), i movimenti descritti da Marks e McAdam (1999) hanno svolto attività di questo tipo presso la sede dell'Unione europea a Bruxelles. In questo tipo di «gioco» poco spazio rimane per la mobilitazione dal basso. Esso infatti è molto distante dalle capacità e dalle inclinazioni delle tipiche basi di riferimento dei movimenti a livello nazionale. La «transnazionalizzazione» della mobilitazione politica pone, infatti, una serie di problemi per lo sviluppo del repertorio d'azione di movimenti sociali. Se la base dei movimenti sociali «pensa globalmente», essa è però costretta ad agire localmente.

L'intervento nelle arene sovranazionali sviluppa invece alcune forme (tipicamente piuttosto moderate) di attivismo vicario, basato su una mobilitazione reale di pochi con un sostegno di limitata entità da parte di molti. Una delle più note TSMOs, Greenpeace, è stata molto creativa nella invenzione di forme di attivismo vicario, capaci di collegare il così detto «cheque-book activism» (cioè l'attivismo di chi si limita ad una sottoscrizione annuale) di una ampia base di sostenitori (circa 4 milioni di persone) con le azioni più radicali di piccoli gruppi di attivisti professionisti. I gommoni di Greenpeace che attirano l'attenzione dell'opinione pubblica sulla strage di balene, le sue mongol- 
fiere che denunciano l'inquinamento causato dal traffico, le sue barche a vela che violano la zona proibita riservata ai test nucleari sottomarini nel Sud Pacifico, o il suo elicottero che atterra su una piattaforma della Shell sono alcuni esempi di questa forma di attivismo. Ancora un'altra forma di «attivismo vicario» è rappresentata da quelli che sono stati definiti come «simulacri di protesta». Ci si è riferiti in particolare a quegli eventi culturali (in particolare i concerti rock) facenti parte di campagne internazionali organizzate dalle TSMOs, che sono frequentati dai partecipanti di base a scopi ludici, ma sono inscenati come eventi della protesta dai loro promotori e interpretati come tali dai mezzi di comunicazione di massa di tutto il mondo (Lahusen 1999).

\section{Movimenti sociali e globalizzazione: qualche conclusione}

In un mondo sempre più globale crescono le tematiche che trascendono le frontiere nazionali: l'internazionalizzazione dei mercati, la ricaduta radioattiva nucleare, l'effetto serra, la distruzione dello strato d'ozono, carestie, povertà, migrazioni internazionali su scala planetaria, la discriminazione delle donne e delle minoranze. Tutti questi sono problemi globali la cui soluzione richiede una cooperazione fra governi nazionali su una scala senza precedenti. Proviamo a riassumere, in questa parte finale, i possibili effetti della globalizzazione sui movimenti.

La globalizzazione porta ad una crescita della quantità di mobilitazione. In primo luogo, essa crea problemi, e quindi conflitti, nuovi, su cui attori collettivi si mobilitano. In secondo luogo, aumenta l'interesse per tematiche «distanti», accrescendo i movimenti di solidarietà. In terzo luogo, l'arena sovranazionale offre una chance in più anche per quei movimenti che, nel loro paese, sono poveri di risorse. Sebbene non abbiano democratic accountability nei termini classici della democrazia rappresentativa, le IGOs sembrano comunque interessate a mantenere dei canali informali di apertura alle organizzazioni di movimento sociale. In queste interazioni, le organizzazioni dei movimenti sociali ricevono dalle IGOs risorse materiali (soprattutto in termini di finanziamenti per progetti specifici) e simboliche (in termini di riconoscimento). Dal canto loro, le IGOs ricevono altre risorse di cui esse hanno bisogno: risorse di lavoro a basso costo da parte delle associazioni di volontariato, ma anche in- 
formazioni e canali di accesso alle popolazioni locali. Al di là dello scambio di risorse sembrano poi emergere talvolta dei fini comuni - come la creazione di norme e istituti sovranazionali.

La globalizzazione accresce, inoltre, le somiglianze tra movimenti sociali di diversi paesi. Canali - formali e informali, diretti e indiretti - promuovono lo scambio di informazioni soprattutto fra paesi che sono geograficamente vicini o che hanno avuto una storia di interazione nel passato. Non solo i movimenti importano da altri movimenti nuove idee e le adattano alla loro realtà, ma anche i governi si copiano l'un l'altro, portando quindi ad un aumento delle somiglianze nei contesti nei quali la protesta ha luogo.

La globalizzazione accentua anche la dimensione sovranazionale dei movimenti. Le interazioni facilitano l'emergere di identità comuni, distaccate dai particolarismi nazionali. Esse inoltre incentivano la creazione di comuni strutture organizzative per la mobilitazione - reticoli transnazionali di organizzazioni di movimenti sociale, campagne transnazionali di protesta.

La globalizzazione tende comunque anche a trasformare le strategie d'azione delle organizzazioni non governative. In generale, i repertori d'azione divengono più moderati, mentre $i$ tentativi di esercitare pressione si spostano dalle piazze alle sedi delle organizzazioni internazionali. Nella costituzione della leadership, le abilità diplomatiche e le informazioni prevalgono rispetto alla manipolazione oratoria o alla capacità di mobilitare le masse. Per quanto riguarda le organizzazioni, abbiamo detto che vi è in atto un processo di routinizzazione. La necessità stessa di essere «riconosciuti» dalle IGOs e la gestione di finanziamenti crescenti comporta l'adozione di statuti interni, basati su regole di accountability formale molto lontane dalla democrazia spontanea e diretta, difesa dai movimenti. La partecipazione a conferenze e riunioni delle IGOs porta ad accettare almeno in parte il principio della delega. L'impegno in interventi «sul campo» in situazioni di emergenza internazionale porta a privilegiare l'efficienza rispetto alla spontaneità. Anche le ideologie divengono più pragmatiche e l'impegno concreto in una realistica strategia di piccoli passi viene preferito rispetto alle utopie delle rivoluzioni globali.

Tutto questo ha portato a chiedersi se ci si trovi ancora davanti a movimenti sociali, oppure se vi sia stata una cooptazione dei leader dei movimenti all'interno delle istituzioni; se i movimenti abbiano adesso più potere, o se i finanziamenti e l'acces- 
so ai luoghi del decision making siano stati pagati con la rinuncia a realizzare trasformazioni effettive. Negli studi sui movimenti sociali, infatti, se alcuni parlano, per riferirsi alla situazione contemporanea, di «società dei movimenti», altri lamentano invece la «fine dei movimenti» (Neidhardt e Rucht 1991; Mayer e Tarrow 1998). Guardando alle azioni delle organizzazioni attive sui temi delle donne, dell'ecologia o dei diritti umani nelle arene sovranazionali si potrebbe infatti essere tentati di sostituire la definizione di «organizzazioni di movimento sociale» con quella, più tradizionale, di «gruppo di interesse pubblico».

Una serie di considerazioni ci portano comunque a sottolineare le differenze (ancora?) esistenti tra movimenti sociali e gruppi di interesse ${ }^{20}$. In primo luogo, le TSMOs di cui abbiamo parlato in questo articolo - seppure burocratizzate nella loro struttura organizzativa - fanno parte di un reticolo più ampio di organizzazioni di movimento, caratterizzate da formule organizzative diverse. Inoltre, pur avendo adottato degli statuti formali, le TSMOs conservano spesso alcuni dei principi della democrazia partecipativa diffusa nei movimenti. Se guardiamo alle TSMOs come membri di un più ampio reticolo, dobbiamo inoltre aggiungere che il lobbying è solo una delle forme d'azione adottate dal movimento delle donne, dal movimento ecologista o dal movimento per i diritti umani di cui le associazioni più formali (e i loro militanti) si sentono parte. E, ancora, molte delle organizzazioni accreditate alle conferenze internazionali delle IGOs non hanno rinunciato ad utilizzare, in altri luoghi, forme d'azione non-convenzionali, seppure prevalentemente non-violente. Infine, se le organizzazioni dei movimenti sociali sono divenute - adeguandosi allo spirito dei tempi - più pragmatiche, la strategia dell'azione esemplare e dei piccoli passi mira spesso ad una trasformazione radicale seppure realizzata attraverso una sensibilizzazione delle coscienze invece che attraverso trasformazioni politiche violente.

La sfida per i movimenti sociali è dunque la capacità di sviluppare strutture organizzative, repertori d'azione e strategie necessarie ad influenzare un sistema di governo a vari livelli che, come abbiamo indicato, offre loro vincoli nuovi ma anche

20 I movimenti sociali si definiscono sulla base di una struttura organizzativa reticolare, una identità collettiva condivisa e riconosciuta dall'esterno, obiettivi di trasformazione sociale e l'uso di repertori d'azione anche non-convenzionali. Per una discussione, cfr. della Porta e Diani (1997, cap. 1). 
nuove opportunità - senza perdere le loro caratteristiche essenziali.

\section{Riferimenti bibliografici}

Almond, G. (1950), The American People and Foreign Policy, New Haven, Yale University Press.

Archer, D. e R. Gartner (1976), Violent Acts and Violent Times: A Comparative Approach to Postwar Homicide Rates, in «American Sociological Review», n. 6, pp. 937-962.

Baglioni, S. (1998), Organizzazioni non governative e conflittualità postmoderne. Il caso delle ONG francesi nella guerra in ex-Jugoslavia, in «Quaderni Forum», n. 11.

Benford, R. e D. Snow (1999), Alternative Types of cross-national Diffusion in the Social Movement Arena, in della Porta, Kriesi e Rucht (a cura di), in stampa.

Blumer, H. (1951), Social Movements, in A. McClung Lee (a cura di), Principles of Sociology, New York, Barnes and Nobles, pp. 199220.

Brysk, A. (1993), From Above and Below. Social Movements, the International System, and Human Rights in Argentina, in «Comparative Political Studies», n. 3, pp. 259-285.

Chatfield, Ch., R. Pagnucco e J. Smith (a cura di) (1997), Solidarity Beyond the State: the Dynamics of Transnational Social Movemen$t s$, Syracuse, Syracuse University Press.

Chilton, P. (1995), Mechanisms of Change: Social Movements, Transnational Coalitions, and the Transformation Processes in Eastern Europe, in Risse-Kappen (a cura di), pp. 189-226.

Collier, D. e R.E. Messick (1975), Prerequisites versus Diffusion: Testing Alternative Explanations of Social Security Adoption, in «American Political Science Review», 69, pp. 1299-1315.

della Porta, D. (1995), Social Movements, Political Violence and the State, Cambridge, Cambridge University Press.

- (1996), Movimenti collettivi e sistema politico, Roma-Bari, Laterza.

- (1998), «1968». Zwischennationale Diffusion und Transnationale Strukturen. Eine Forschungsagenda, in «Geschichte und Gesellschaft», n. 17, n. speciale su «1968», pp. 131-150.

della Porta, D. e M. Diani (1997), I movimenti sociali, Roma, Carocci. della Porta D., H. Kriesi e D. Rucht (a cura di) (1999), Social Movements in a Globalizing World, New York, Macmillan, in stampa.

della Porta, D. e H. Reiter (a cura di) (1998), Policing Protest. The Control of Mass Demonstration in Contemporary Democracies, Minneapolis, University of Minnesota Press.

della Porta, D. e D. Rucht (1995), Social Movement Sectors in Context: 
A Comparison of Italy and West Germany, 1965-1990, in J.C. Jenkins e B. Klandermans (a cura di), The Politics of Social Protest, Minneapolis, University of Minnesota Press, pp. 229-272.

Duyvendak, J.-W. e R. Koopmans (1995), The Political Construction of the Nuclear Energy Issue, in H. Kriesi, R. Koopmans, J.W. Duyvendak e M.G. Giugni, New Social Movements in Western Europe. A Comparative Analysis, Minneapolis, University of Minnesota Press, pp. 145-164.

Eberwein, W.-D. (1997), Die Politik Humanitärer Hilfe: Im Spannungsfeld von Macht und Moral, WZB Discussion Paper n. P97-301.

Eisinger, P.K., (1973), The Protest of Protest Bebavior in American Cities, in «American Political Science Review», n. 1, pp. 11-28.

Garrett, G. e P. Lange (1995), Internationalization, Institutions, and Political Change, in «International Organization», n. 4, pp. 627655.

Giddens, A. (1990), The Consequences of Modernity, Cambridge, Polity Press.

- (1994), Brave New World: The New Context of Politics, in D. Miliband (a cura di), Reinventing the Left, Cambridge, Polity Press, pp. 21-38.

Giugni, M.G. (1995), The Cross-National Diffusion of Protest, in Kriesi, Koopmans, Duyvendak e Giugni (a cura di), New Social Movements in Western Europe. A Comparative Analysis, Minneapolis, University of Minnesota Press, pp. 181-206.

Goertz, G. (1994), Contexts of International Politics, Cambridge, Cambridge University Press.

Haas, P.M. (1992), Introduction, in P.M. Haas (a cura di), Knowledge, Power and International Policy Coordination, A Special Issue of «International Organization», n. 1, pp. 1-36.

Hall, P. (1993), Policy Paradigms, Social Learning, and the State. The Case of Economic Policymaking in Britain, in «Comparative Politics», n. 2, pp. 275-296.

Holthaus, I. (1996), Neuere Entwicklungen der internationalen Frauenbewegung: Reaktionen auf globale Prozesse, in «Forschungsjournal Neue Soziale Bewegungen», n. 2, pp. 61-70.

Hooghe, L. e G. Marks (1995), Birth of a Polity: The Struggle over European Integration, in Kitschelt, Lange, Marks e Stephens (a cura di), The Politics and Political Economy of Advanced Industrial Societies, Cambridge, Cambridge University Press.

Imig, D. e S. Tarrow (1999), The Europeanization of Movements? Contentious Politics and the European Union. October 1983-March 1995, in della Porta, Kriesi e Rucht (a cura di), in stampa.

Janett, D. (1996), Allianzsysteme von Nicht-Regierungsorganisationen in der Klimapolitik, in «Forschungsjournal Neue Soziale Bewegungen», n. 2, pp. 83-89.

Karl, T.L. e Ph.C. Schmitter (1994), Democratization around the Glo- 
be: Opportunities and Risks, in M.T. Klare e D.C. Thomas (a cura di), World Security: Challenges for a New Century, New York, St. Martin's Press, pp. 43-62.

Klandermans, B., M. de Weerd, M. Sabucedo e J.-M. Sabucedo (1999), Injustice and Adversarial Frames in a Supranational Political Context: Farmer's Protest in the Netherlands and Spain, in della Porta, Kriesi e Rucht (a cura di), in stampa.

Knopf, J.W. (1993), Beyond Two-level Games: Domestic-international Interaction in the Intermediate Range Nuclear Forces Negotiations, in «International Organization», 47, pp. 599-628.

Koopmans, R. (1999), Globalization or Still National Politics? A Comparison of Protests against the Gulf War in Germany, France, and the Netherlands, in della Porta, Kriesi e Rucht (a cura di), in stampa.

Kriesi, H. (1991), The Political Opportunity Structure of New Social Movements: Its Impact on their Mobilization, FS III 91-103, Berlin, Wissenschaftszentrum.

Lahusen, Ch. (1999), International Campaigns in Context: Collective Action Between the Local and the Global, in della Porta, Kriesi e Rucht (a cura di), in stampa.

Leary, V. (1979), A New Role for Non-Governmental Organizations in Human Rigbts: A Case Study of Non-Governmental Participation in the Development of International Norms on Torture, in A. Cassese (a cura di), UN Law/Fundamental Rights: Two Topics in International Laws, The Netherlands, Sijthaff and Noordhoff, pp. 197-210.

LeSaout, D. (1995), Mobilisations protestataires et gestion du sens. La mobilisation contre le Cruise et Pershing II. Tesi di dottorato, Firenze, Istituto Universitario Europeo.

Lipsky, M. (1965), Protest in City Politics, Chicago, Rand McNally \& C.

Luthardt, W. (1993/4), European Integration and Referendums, in T. Ellwein et al., Jabrbuch zur Staats- und Verwaltungswissenschaft, vol. 6, Baden-Baden, Nomos-Verlagsgesellschaft, pp. 209-233.

Marks, G. e D. McAdam (1999), On the relationship of the Political Opportunities to the Form of Collective Action, in della Porta, Kriesi e Rucht (a cura di), in stampa.

Marks, G., F. Nielsen, L. Ray e J.E. Salk (1996), Competencies, Cracks, And Conflicts. Regional Mobilization in the European Union, in «Comparative Political Studies», n. 1, pp. 164-192.

Marx Ferree, M. e W.A. Gamson (1999), The Gendering of Abortion Discourse: Assessing Global Feminist Influence in the United States and Germany, in della Porta, Kriesi e Rucht (a cura di), in stampa.

McAdam, D. (1996), 'Initiator' and 'Derivative' Movements: Diffusion Processes in Protest Cycles, in M. Traugott (a cura di) Repertoires and Cycles of Collective Action, Durham, N.C., Duke University Press, pp. 217-239. 
McAdam, D. e D. Rucht (1993), The Cross-national Diffusion of Movement Ideas, in «The Annals of the AAPSS», n. 528, pp. 56-74.

McAdam, D., S. Tarrow e Ch. Tilly (1996), To Map Contentious Politics, in «Mobilization», 1, pp. 17-34.

McCarthy, J.D., C. McPhail e J. Crist (1999), The Diffusion of Public Order Management Systems: Protest Cycles and Official Responses, in della Porta, Kriesi e Rucht (a cura di), in stampa.

McGrew, A.G. (1992), Conceptualizing Global Politics, in A.G. McGrew e P.G. Lewis, Global Politics. Globalization and the NationState, Cambridge, Polity Press, pp. 1-28.

Marquis, D. (1997), Politique extérieure et démocratie directe. Effets du consensus et du conflit sur la formation des attitudes en matière de politique extérieure suisse, Université de Génève, Département de science politique, Etudes et Recherche, n. 35.

Mayer, D.S. e S. Tarrow (a cura di) (1998), The Social Movement Society, New York, Rowan and Littlefield.

Neidhardt, F. e D. Rucht (1991), The Analysis of Social Movements: The State of the Art and Some Perspective for Further Research, in D. Rucht (a cura di), Research in Social Movements: The State of the Art, Boulder, Westview Press, pp. 421-464.

Pagnucco, R. (1995), The Comparative Study of Social Movements and Democratization: Political Interaction and Political Process Approaches, in «Research in Social Movements, Conflict and Change», 18 , pp. 145-183.

Passy, F. (1999), Supranational Political Opportunities as a Channel of Globalization of Political Conflicts: The Case of the Rights of Indigenous People, in della Porta, Kriesi e Rucht (a cura di), in stampa.

Pitcher, B.L., R.L. Hamblin e J.L. Miller (1978), The Diffusion of Collective Violence, in «American Sociological Review», n. 1, pp. 23 35.

Princen, T. (1994), NGOs: Creating a Niche in Environmental Diplomacy, in Princen e Finger (a cura di), pp. 29-47.

- (1995), Ivory, Conservation, and Environmental Transnational Coalitions, in Risse-Kappen (a cura di), pp. 227-253.

Princen, T. e M. Finger (a cura di) (1994a), Environmental NGOs in World Politics. Linking the Local and the Global, London-New York, Routledge.

Princen, T. e M. Finger (1994b), Introduction, in Princen e Finger (a cura di), pp. 1-25.

Putnam, R.D. (1988), Diplomacy and Domestic Politics: The Logic of Two-level Games, in «International Organization», n. 3, pp. 427-60.

Risse-Kappen, T. (1995b), Bringing Transnational Relations Back In: An Introduction, in Risse-Kappen (a cura di), pp. 3-33.

Risse-Kappen, T. (a cura di) (1995a), Bringing Transnational Relations Back In, Cambridge, Cambridge University Press. 
Robertson, R. (1992), Globalization. Social Theory and Global Culture, London, Sage.

Rochon, T.R. (1988), Mobilizing For Peace, Princeton, Princeton University Press.

Roggeband, C. (1997), Cross-national Diffusion of Social Movement Ideas and Practices. The Spread of Feminism Across Countries, paper presentato alla Summer School on Social Movements, Rovaniemi, giugno.

Rosenau, J.N. (1990), Turbulence in World Politics. A Theory of Change and Continuity, New York, Harvester Wheatsheaf.

Rucht, D. (1993), 'Think Globally, Act Locally'? Needs, Forms and Problems of Cross-National Cooperation Among Environmental Groups, in J.D. Liefferink, P.D. Lowe e A.P.J. Mol (a cura di), European Integration and Environmental Policy, London, Belhaven Press, pp. 75-95.

Rucht, D. (1997), Environmental Policy for the European Community: Problems of Mobilizing Influence in Brussels, in Chatfield, Pagnucco e Smith (a cura di).

Schaefer Caniglia, E. (1995), Environmental Transnational Social Movement Organizations: Balancing Power in International Policy Making, paper presentato alla American Sociological Association Annual Conference, Washington, 19-23 agosto.

Schmitter, Ph.C. (1993/94), Representation and the Future Euro-Polity, in T. Ellwein et al., Jabrbuch zur Staats-und Verwaltungswissenschaft, vol. 6, Baden-Baden, Nomos-Verlagsgesellschaft, pp. 55-81.

- (in stampa), Imagining the Future of the Euro-Polity with the Help of New Concepts, in G. Marks, Ph. Schmitter e W. Streeck, The Emerging Euro-Polity: Its Powers, Policies and Prospects, London, Sage.

Sharoni, S. (1995), Gender and the Israeli-Palestinian Conflict: The Politics of Women Resistence, Syracuse, Syracuse University Press.

Sikkink, K. (1993), Human Rights, Principled Issue-networks, and Sovereignty in Latin America, in «International Organization», n. 3, pp. 411-441.

Smith, J. (1995), Transnational Political Processes and the Human Rights Movement, in «Research in Social Movements, Conflict and Change», 18, pp. 187-221.

- (1999), Global Politics and Transnational Campaign Against International Trade in Toxic Waste, in della Porta, Kriesi e Rucht (a cura di), in stampa.

Smith, J., R. Pagnucco e W. Romeril (1994), Transnational Social Movement Organisations in the Global Political Arena, in «Voluntas», n. 2, pp. 121-154.

Spilerman, S. (1976), Characteristics and Severity of Racial Disorders, in «American Sociological Review», n. 5, pp. 771-792.

Stark, D. e L. Bruszt (1990), Negotiating the Institutions of Democracy: 
Contingent Choices and Strategic Interactions in the Hungarian and Polish Transitions, Working Papers On Transitions from State Socialism, Mario Einaudi Center for International Studies, Cornell University.

Starr, H. (1991), Democratic Dominoes. Diffusion Approaches to the Spread of Democracy in the International System, in «Journal of Conflict Resolution», n. 2, pp. 356-381.

Strang, D. e J.W. Meyer (1993), Institutional Conditions for Diffusion, in «Theory and Society», n. 4, pp. 487-511.

Tarrow, S. (1995), The Europeanization of Conflict: Reflections from a Social Movement Perspective, in «West European Politics», n. 2, pp. 223-251.

Tilly, Ch. (1984), Social Movements and National Politics, in Ch. Bright e S. Harding (a cura di), Statemaking and Social Movements: Essays in History and Theory, Ann Arbor, University of Michigan Press, pp. 297-317.

Traxler, F. e Ph.C. Schmitter (1995), The Emerging Euro-Polity and Organized Interests, in «European Journal of International Relations», n. 2, pp. 191-218.

Turner, R. e L. Killian (1987), Collective Behavior, Englewood Cliffs, Prentice Hall.

Walk, H. e A. Brunnengräber (1996), Ad-boc-Allianzen - eine neue gesellschaftspolitische Perspektive?, in «Forschungsjournal Neue Soziale Bewegungen», n. 2, pp. 70-82.

Walker, J.L. (1969), The Diffusion of Innovations among the American States, in «American Political Science Review», n. 3, pp. 880-899.

Wallerstein, I. (1990), Antisystemic Movements. History and Dilemmas, in S. Amin, G. Arrighi, E.G. Frank e I. Wallerstein, Transforming the Revolution. Social Movements and the World-System, New York, Monthly Review Press, pp. 13-54.

Wapner, P. (1995), Politics Beyond the State. Environmental Activism and the World Civic Politics, in «World Politics», n. 3, pp. 311340.

- (1996), Environmental Activism and World Civic Politics, Albany, State University of New York Press.

Wisler, D. (1994), Violence politique et mouvements sociaux, Génève, Editions Georg. 